\title{
miRNA-148b-3p targeting SOCS3 inhibits macrophage M2 polarization by JAK2/STAT3 pathway in immune thrombocytopenia
}

\author{
Yang YANG; Lijuan FU; Chunmei CHEN; Meiwei HU* \\ Department of Hematology, The Second Hospital Affiliated to Zhejiang Chinese Medical University, Hangzhou, 310005, China
}

Key words: ITP, miR-148b, Macrophage, SOCS3

\begin{abstract}
Aberrant expression of miRNAs is significantly correlated with the occurrence of immune thrombocytopenic purpura (ITP). The immune imbalance of M1/M2 macrophage contributes to the development of ITP. However, the role of miR-148b-3p in macrophage phenotype imbalance remains unknown in ITP. In this study, we aimed to explore whether miR-148b-3p inhibits M2 macrophage polarization in ITP and to investigate the underlying mechanism. Peripheral blood from 22 ITP patients were collected, and real-time PCR confirmed that miR-148b-3p was upregulated and Western blot analyses detected the expression of SOCS3 was down-regulated. Subsequent dualluciferase reporter gene assay indicated that miR-148b-3p could bind to SOCS3. Furthermore, we found significant correlation between miR-148b-3p expression and platelet count. Applying gain and lose the function experiments of miR-148b-3p and SOCS3, we demonstrated that suppression of miR-148b-3p or up-regulation of SOCS3 promoted macrophage M2 polarization by inhibiting JAK2/STAT3 pathway. Together, our findings demonstrate that that miR148b-3p targeting SOCS3 inhibits M2 macrophage polarization via JAK2/STAT3 signaling in ITP.
\end{abstract}

\section{Introduction}

Immune thrombocytopenic purpura (ITP) is a common autoimmune disease, which is resulted in a low platelet count and bleeding in the majority of patients. The main mechanisms include decreased platelet production and autoantibody-against platelet demolition (Cines et al., 2009). During this process, $\mathrm{T}$ cell dysfunction, such as regulatory $\mathrm{T}$ cells (Treg) imbalance, and abnormal activation of cytotoxic $\mathrm{T}$ cells are considered to play a prominent role (Yu et al., 2015). However, accumulating evidence indicates that the aberrant differentiation and function of macrophage are also essential in the pathogenesis of ITP (Chi et al., 2019).

Macrophages are highly heterogeneous cells that can be activated by environmental stimuli and polarized to functionally different phenotypes. Macrophages can mainly differentiate into two subsets, classically activated M1 or alternatively activated M2 phenotype macrophages (Wang et al., 2014, Cui et al., 2018). M1 polarization is characterized by their ability to produce relatively high levels of proinflammatory cytokines, such as interleukin-1 (IL-1), IL-6, IL-12 and tumor necrosis factor- $\alpha$ (TNF- $\alpha$ ), and promote an inflammatory response (Joshi et al., 2010, Batra et al., 2018).

*Address correspondence to: Meiwei Hu, humweiwei@sina.cn Received: 14 January 2021; Accepted: 11 April 2021
In contrast, M2 macrophages mediate immunoregulatory functions through express anti-inflammatory cytokines, such as IL-10, Arg-1 and transforming growth factor- $\beta$ (TGF- $\beta$ ) (Liu et al., 2020). Coordination of the macrophage M1/M2 polarization could impact the balance of lymphocyte subset differentiation and change their tolerogenic scavenging function, hence facilitating to the pathogenesis of autoimmune diseases. However, current studies about aberrant macrophage polarization are limited, and the role and deep-going regulatory mechanism in ITP remain to be elucidated.

MicroRNAs (miRNAs) are small non-coding RNAs involved in the regulation of biological processes at the posttranscriptional level. The dysregulation of miRNAs contributes to many pathological processes that involve the immune system, such as cancer and autoimmunity (Pauley et al., 2009, Singh et al., 2013). Meanwhile, increasing evidence illuminated that aberrant expression of many miRNAs have been discovered in ITP implying that miRNAs are likely playing a prominent role in the pathogenesis of ITP. miR-99a, miR-182-5p, miR-183-5p and miR-125a-5p expression levels were abnormal and those miRNAs contributed to induce immune imbalance of Treg/Th17 in ITP (Li et al., 2016, Hua et al., 2019). Besides, silencing of miR-155-5p could initiate the PD1/PDL1 pathway-mediated macrophage M2 polarization and inhibit ITP progression by up-regulating SOCS1 (Chang et al., 2020). Given this 
background we examined differences in the proportion of M2 phenotype macrophages in ITP patients and healthy controls and tested the hypothesis that miR-148b-3p regulates the development of ITP via the JAK2/STAT3 pathway via modulating the expression of SOCS3. In this study, peripheral blood mononuclear cells (PBMCs) were collected and induced into macrophages. Then, we profiled the expressions of miR-148b-3p in cells and identified miR-148b-3p as a candidate miRNA that promotes progression of ITP. We thus performed further gain- and loss-of-function studies to demonstrate the role of miR-148b-3p and SOCS3 in the development of ITP in vitro.

\section{Materials and Methods}

\section{Patients}

We enrolled 22 patients with confirmed ITP who were admitted to The Second Affiliated Hospital of Zhejiang Chinese Medical University from June 2017 to June 2018 (Suppl. Table S1). Meanwhile, we enrolled 15 healthy subjects and all controls had a normal platelet count and did not receive any steroid therapy. There was no significant difference in age and sex between patients with ITP and healthy controls. Patients with ITP were diagnosed according to a report from the American Society of Hematology, cases with diabetes, hypertension, cardiovascular diseases, pregnancy, active or chronic infection, connective tissue diseases were excluded from this study. Bone marrow aspirate was performed to exclude other diseases resulting in thrombocytopenia. The protocols of this study were approved by the Ethics Committee of The Second Affiliated Hospital of Zhejiang Chinese Medical University (2019-KL-049-01) and were based on the ethical principles of the Declaration of Helsinki. Written informed consent was obtained from all patients prior to the study.

Isolation of CD14+ monocyte cells and induction of macrophages

Peripheral blood was collected from patients with ITP. PBMCs were isolated from peripheral blood using Ficoll reagent (GE Healthcare, USA) by density gradient centrifugation (Qian et al., 2015). CD14+ monocyte cells were isolated from PBMCs using CD14 MicroBeads Kit (Miltenyi Biotec, USA) according to the manufacturer's instructions. Monocyte cells were incubated in RPMI 1640 medium supplemented with $10 \%$ fetal bovine serum (FBS) for 7 days, and half of the medium renewed every 2 days. The cells were added with $200 \mathrm{ng} / \mathrm{ml}$ Phorbol 12-myristate 13-acetate (PMA) and incubated for another 3 days, whereupon macrophage phenotype was induced (Park et al., 2007).

\section{Cell transfection}

For transfection, the macrophages induced by PMA/IL-4 were plated in 6 well plate at a cell density of $2 \times 105$ cells/well. Negative Control (NC) mimic (5'-UUCUCCGAACGUGUCACGUTT-3'), miR-148b-3p mimic (5'-UUAAUG CUAAUCGUGAUAGGGGU-3'), NC inhibitor (5'-CAGUACUUUUGUGUAGUACAA-3'), miR-148b-3p inhibitor (5'-ACCCCUAUCACGAUUAGCAUUAA-3'), NC-plasmid, SOCS3-plasmid
(OriGene Global, USA) were transfected into cells using Lipofectamine 2000 reagent (Thermo Fisher Scientific, USA) according to the manufacturer's instructions. After $48 \mathrm{~h}$ incubation, the cells were harvested for subsequent experiments.

\section{Flow cytometry and ELISA}

Macrophages transfected miR-148b-3p inhibitor and SOCS3plasmid were collected and blocked in 3\% BSA in PBS for $30 \mathrm{~min}$. Then cells were reacted with PE-anti-CD206 antibody (BioLend, USA) in the dark and subjected to flow cytometry. M2 macrophages were defined as CD206+ cells.

The levels of IL-10 and TGF- $\beta$ in macrophages culture supernatants were measured by ELISA kits (R\&D Systems, USA) according to the manufacturer's instructions. All samples were tested in triplicate.

\section{Quantitative real-time RCR (RT-qPCR) and Western blot}

Total RNAs from monocytes under different treatments were extracted using TRIzol reagent (Thermo Fisher Scientific, USA), and reversely transcribed into cDNA based on the manufacturer's protocol of a cDNA kit (Takara, Japan). RTqPCR was conducted according to the instructions of SYBR Green Master Mix (Takara, Japan) for the detection of miR148b-3p (F:5'-TCTCTCCAGTCTACTCAGTGCATC-3', R: 5'-TATGGTTTTGACGACTGTGTGAT-3') and SOCS3 expression (F: 5'-AGCAGCGATGGAATTACCTGGAAC-3', R: 5'-TCCAGCCCAATACCTGACACAGAA-3';). The relative expression was calculated with the formula $2-\Delta \Delta \mathrm{ct}$, with U6 (F: 5'-CTCGCTTCGGCAGCACA-3', R: 5'-AACGCTTCACGAATTTGCGT-3') or GAPDH (F: 5'-CGAACCTCTCTGCTCCTCCTGTTCG-3'， R: 5'-CATGGTGTCTGAGCGATGTGG-3') as internal references.

The total protein was extracted from the cells with RIPA Buffer (CST, USA), and protein concentrations were determined using a BCA Protein Assay Kit (CST, USA). A total of $30 \mu \mathrm{g}$ protein sample was electrophoresed on $10 \%$ sodium dodecyl sulfate polyacrylamide gel electrophoresis (SDS-PAGE) and transferred onto polyvinylidene fluoride membrane (PVDF). After blocking with 5\% fat-free milk, the membranes were incubated at $4^{\circ} \mathrm{C}$ overnight with the following primary antibodies: anti-SOCS3 (ab236519, Abcam), anti-CD206 (91992S, CST), anti-JAK2 (3230S, CST), anti-p-JAK2 (3771S, CST), anti-STAT3 (9139S, CST), anti-p-STAT3 (9145S, CST) and GAPDH (5174S,CST), and then incubated with secondary antibody conjugated to horseradish peroxidase (HRP) (CST, USA) at room temperature for $2 \mathrm{~h}$. The blots were visualized with ECL kit and band gray value was analyzed with Quantity One software (Bio-Rad, USA). GAPDH was detected and acted as control protein.

\section{Dual-luciferase reporter gene assay}

Wild type sequence of SOCS3 (SOCS3-WT) and its mutant sequence (SOCS3-MUT) were inserted into pmirGLO luciferase reporter (Promega, USA), respectively. Then, 293 $\mathrm{T}$ cells were co-transfected with miR-148b-3p mimic and SOCS3-WT or SOCS3-MUT plasmids using Lipofectamine 2000 reagent. The relative luciferase activity was measured with the Dual-Luciferase reporter assay system (Promega, USA) according to the manufacturer's protocol. 


\section{Statistical analysis}

Statistical analyses were done using SPSS19.0 software and GraphPad Prism software. Data was expressed as mean SD. The difference of means between two groups was tested by independent samples $T$-test. Pearson analysis was used to analyze correlation between indicators. The level of significance was set to $P$-value less than 0.05 .

\section{Results}

miR-148b-3p is up-regulated in peripheral blood of patients with ITP

Previous studies reported that the dysregulation of miRNAs is important in the development of ITP, especially in monocytes. We performed analysis five interested miRNAs in 22 ITP patients and 15 healthy controls. Among them, miR-148b3 p was significantly upregulated in ITPs (Suppl. Fig. S1). In addition, the study of miR-148b-3p was rarely reported in ITPs. Relative miR-148b-3p expression was higher in monocyte cells from ITP patients than in those from controls (Fig. 1A). Furthermore, we found a negative correlation between miR-148b-3p expression levels and platelet counts (Fig. 1B, $\mathrm{r}=-0.7628, P<0.01$ ). Compared with healthy controls, CD206 expression was significantly downregulated in ITP determined by flow cytometry (Fig. 1C, $P<0.01$ ). These results indicated that upregulation of miR-148b-3p could is significantly correlated with ITP by regulating M2 macrophages.

Suppression of $m i R-148 b-3 p$ promotes macrophage M2 polarization and inhibits the occurrence of ITP

To investigate the effect of miR-148b-3p on ITP via macrophage M2 polarization, we extracted PBMCs from peripheral blood of ITP patients and then CD14+ monocyte cells were separated with anti-CD14 microbeads, and monocytes cells induced macrophages through PMA
(Fig. 2A). Then, cells were introduced with NC inhibitor and miR-148b-3p inhibitor. The miR-148b-3p expression was obviously decreased in CD14+ cells of the miR-148b-3p inhibitor group, indicating the infection of miR-148b-3p inhibitor was worked (Fig. 2B). In addition, the results of Western blot analysis showed that the protein expression of CD206 in the miR-148b-3p inhibitor-treated cells was significantly up-regulated (Fig. 2C). Consistently, flow cytometry analysis displayed that the percentage of CD206+ M2 macrophages was appreciably increased (Fig. 2D). Afterwards, ELISA results demonstrated that the concentrations of IL-10 and TGF- $\beta$ were elevated in the miR$148 \mathrm{~b}-3 \mathrm{p}$ inhibitor group compared with NC group (Fig. 2E). These findings suggested that down-regulation of miR-148b-3p could promote macrophage M2 polarization in ITP.

\section{miR-148b-3p promotes ITP via targeting and down-regulating} SOCS3

To clarify whether there was a targeting relationship between miR-148b-3p and SOCS3, we utilized TargetScanHuman 7.2 software to predict that the sequence of SOCS3 harbors the binding site of miR-148b-3p (Figs. 3A and 3B). Then, dualluciferase reporter gene assay was performed in $293 \mathrm{~T}$ cells. Luciferase reporter assay displayed that miR-148b-3p mimic caused obvious decrease in luciferase activity in SOCS3-WT reporter compared with the mimic NC group, whereas had no noticeable effect on the luciferase activity in SOCS3MUT reporter (Fig. 3C). In addition, miR-148b-3p overexpression with mimic significantly inhibited SOCS3 expression at both mRNA and protein levels (Figs. 3D and $3 \mathrm{E})$. These analyses suggested that miR-148b-3p could target SOCS3 and suppress the expression of SOCS3. Meanwhile, expression of SOCS3 was lower in CD14+ monocyte cells from ITP patients than in cells from controls (Fig. 3F), and a negative correlation between SOCS3 and miR-148b-3p expression levels was found (Fig. 3G). These findings
(A)

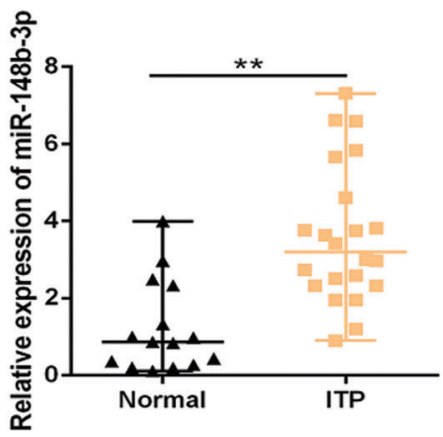

(C)

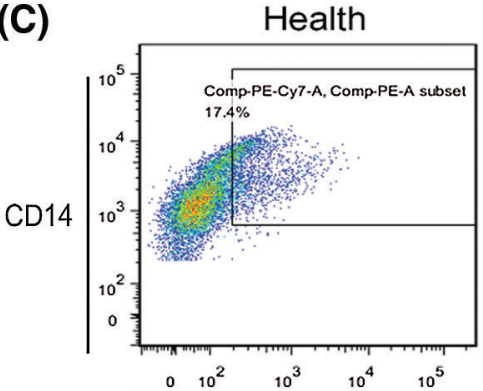

(B)

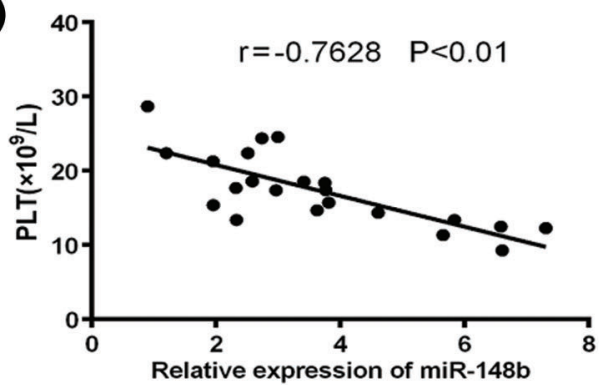

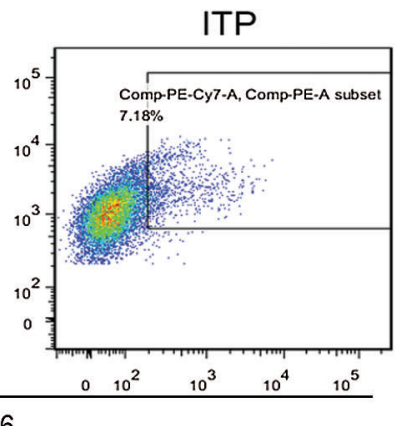

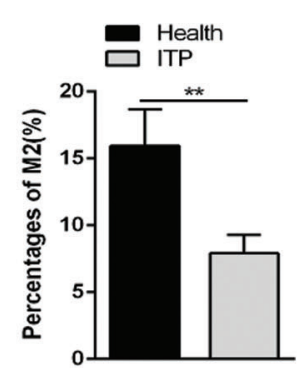

FIGURE 1. Relative expression of miR-148b expression in ITP patients $(\mathrm{N}=22)$ and controls $(\mathrm{N}=15)$. (A) Relative expression of miR-148b in CD14+ cells from ITP patients and healthy controls. ${ }^{* *} P<0.01$ vs. control group. (B) Correlation analysis among miR-148b and platelet counts. $r=-0.7628,{ }^{*} P<0.01$. (C) Percentages of macrophage M2 were compared by flow cytometry. ${ }^{* *} P<$ 0.01 vs. control group. 
(A)
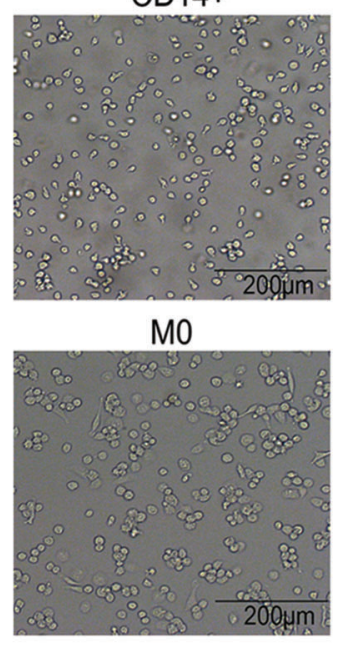

(B)

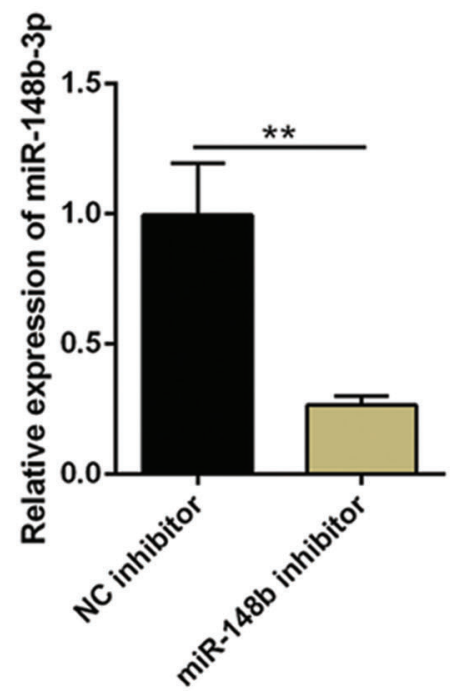

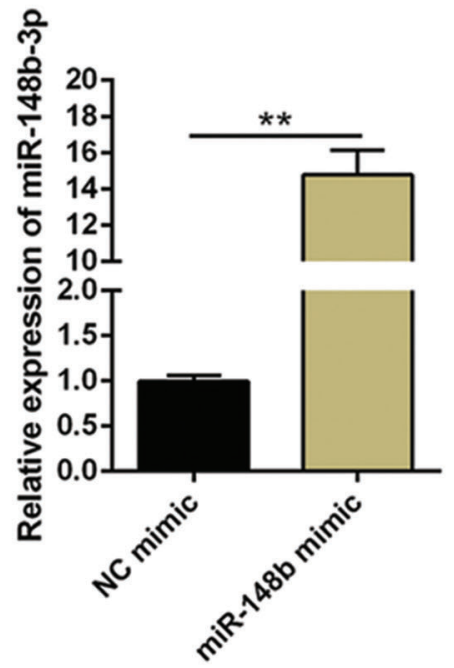

(C)
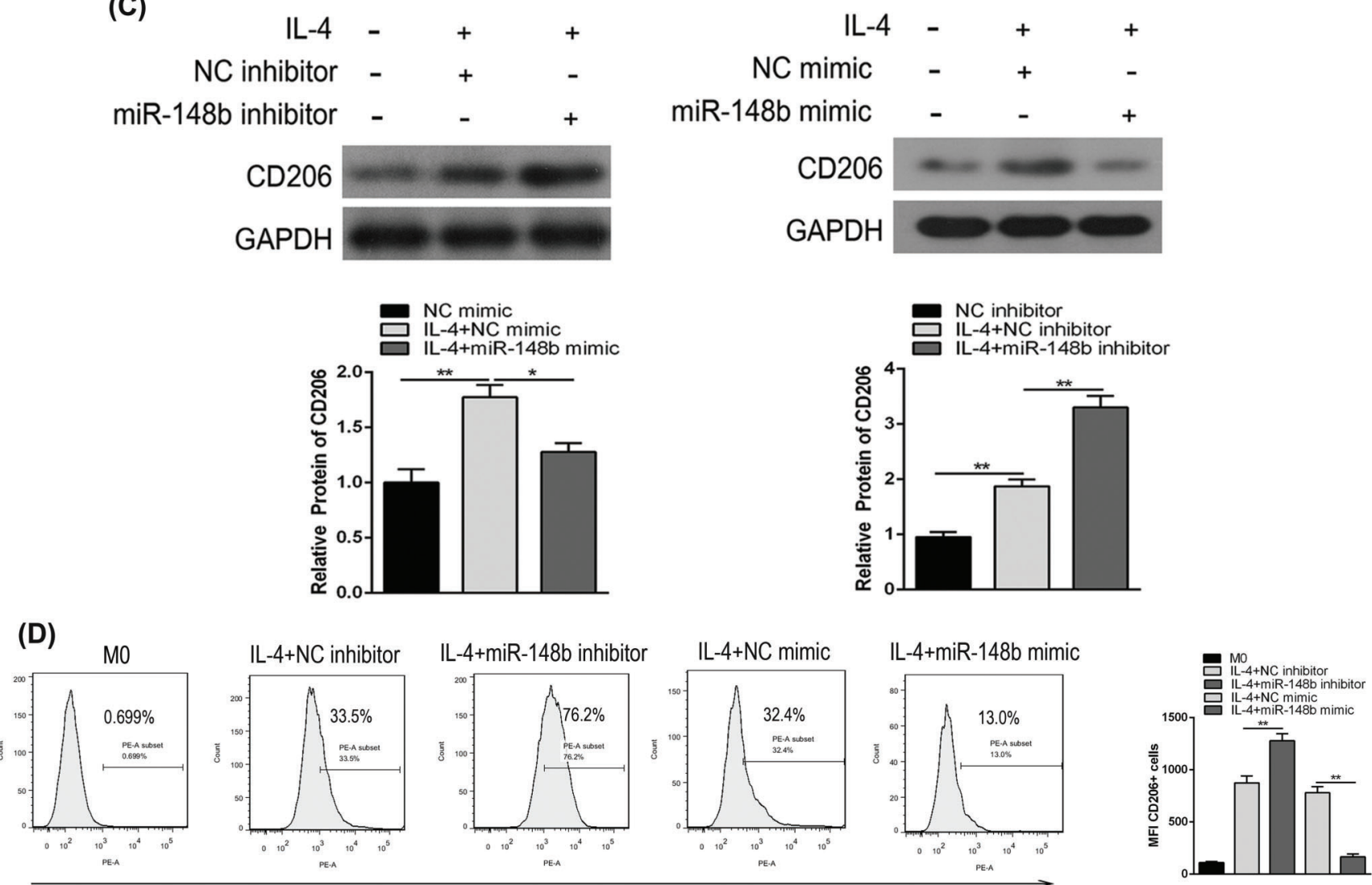

CD206
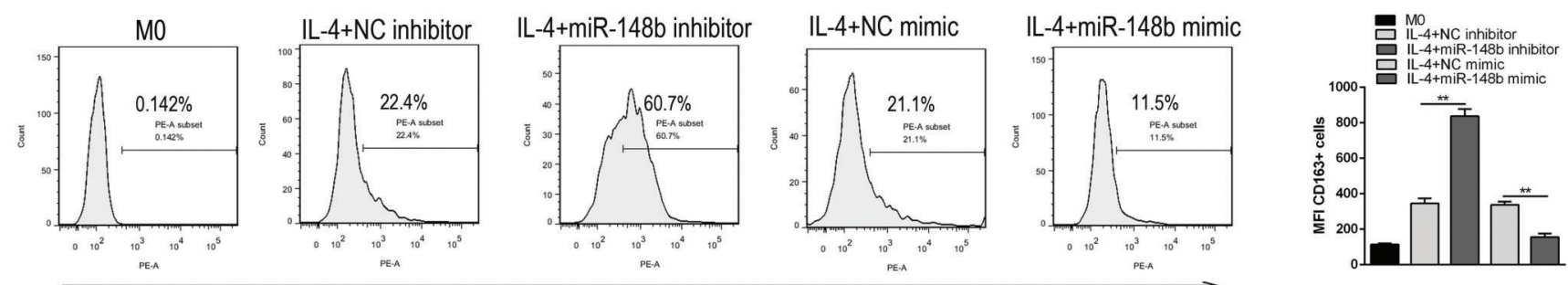

FIGURE 2. (continued) 

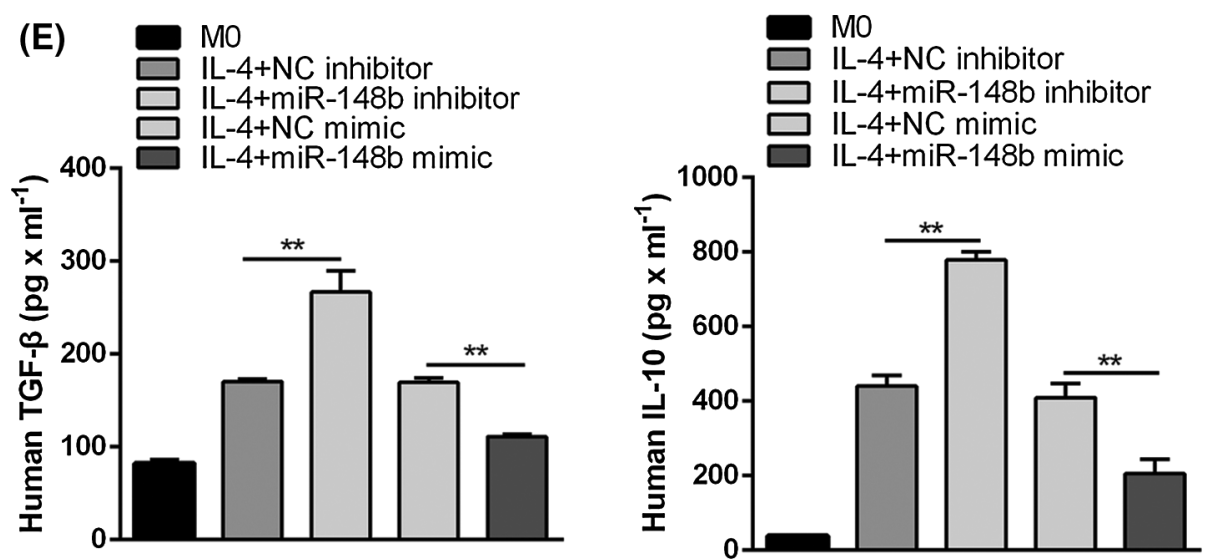

FIGURE 2. Suppression of miR-148b promotes macrophage M2 polarization. (A) Representative microscopic images of macrophage M0 morphology $(\times 200)$. (B) The expression of miR-148b was regulated by inhibitor and mimic in macrophages. $P<0.01$ vs. NC. (C-D) Macrophage M2 marker CD206 protein expression respectively assessed by Western blot analysis and flow cytometry. (E) IL-10 and TGF$\beta 1$ expression measured by ELISA.

(A)

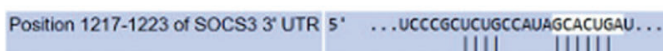

hsa-miR-148b-3p 3. UguUucaAgacacua-CGUGaCU

(B) SOCS3MUT1 5' UCCCGCUCUGCCAUACGUGACAU 3'

SOCS3 WT1 5' UCCCGCUCUGCCAUAGCACUGAU 3'

hsa-miR-148b-3p 3' UGUUUCAAGACACUACGUGACU 5'
(C)

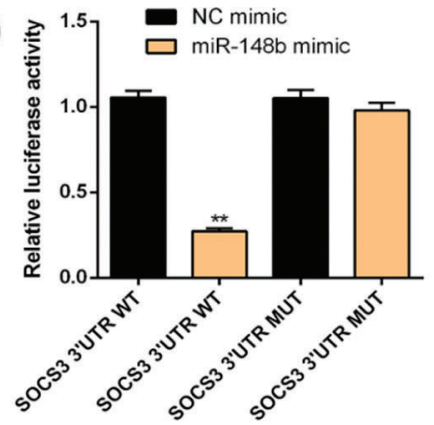

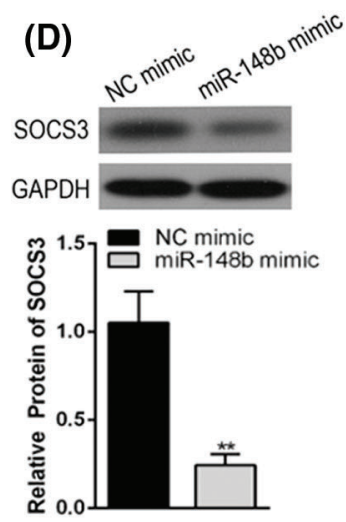
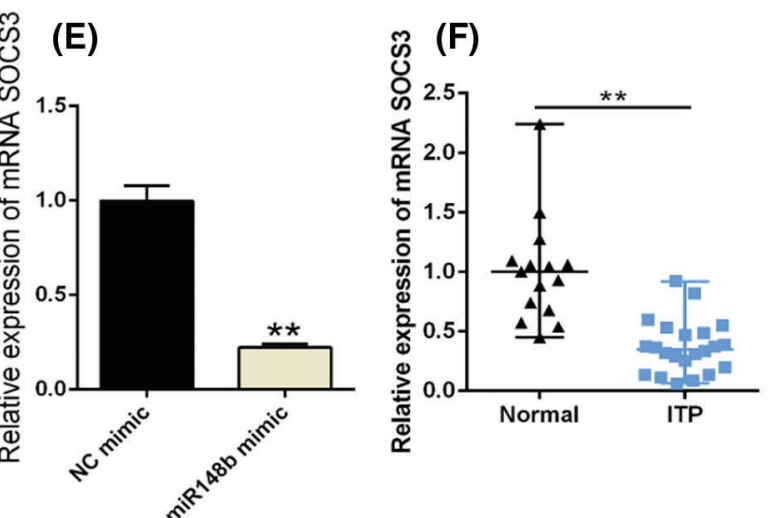

(G)

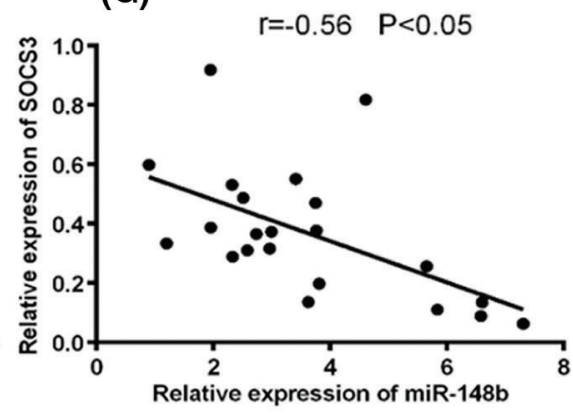

FIGURE 3. SOCS3 is a direct target of post-transcriptional repression by miR-148b-3p. (A-B) The predicted binding site for miR-148b-3p of the SOCS3 3'UTR. (C) Luciferase reporter assay was conducted in $293 \mathrm{~T}$ cells. Data are presented as averages from three independent experiments. (D-E) The mRNA and protein levels of SOCS3 were detected by RT-qPCR and western blot. (F) Relative expression of SOCS3 in CD14+ cells from ITP patients and healthy controls. ${ }^{*} P<0.01 v s$. control group. (G) Correlation analysis among SOCS3 and platelet counts. $\mathrm{r}=-0.56,{ }^{\star} P<0.05$.

indicated that miR-148b-3p could affect the occurrence of ITP by targeting SOCS3.

SOCS3 overexpression reverses the miR-148b-3p mediated inhibition of M2 polarization

We displayed above the upregulation of SOCS3 in monocyte cells of ITP patients. To explore the regulatory mechanisms of SOCS3 on macrophage polarization, miR-148b-3p mimic and SOCS3 plasmid were transfected into macrophage cells. Satisfactory transfection efficiency was obtained after $48 \mathrm{~h}$ (Figs. 4A and 4B). Flow cytometry analysis showed that the percentage of CD206+ macrophages was significantly reversed in the SOCS3 + miR-148b-3p mimic group compared with the SOCS3 + mimic NC group (Fig. 4C), whereas western blot analysis revealed the protein expression of SOCS3 was consistent with CD206 (Fig. 4F). Meanwhile, ELISA results indicated that the concentrations of IL-10 and TGF- $\beta$ were increased in the 
(A)

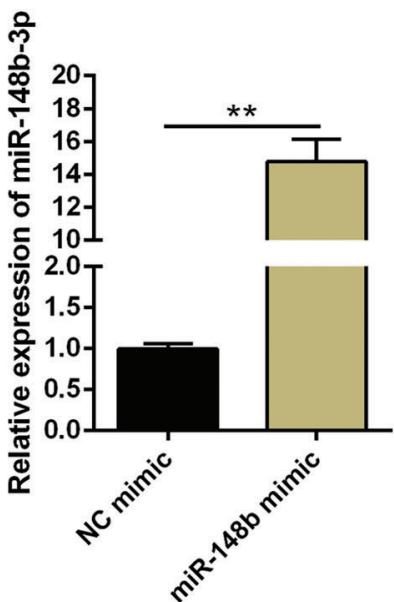

(B)

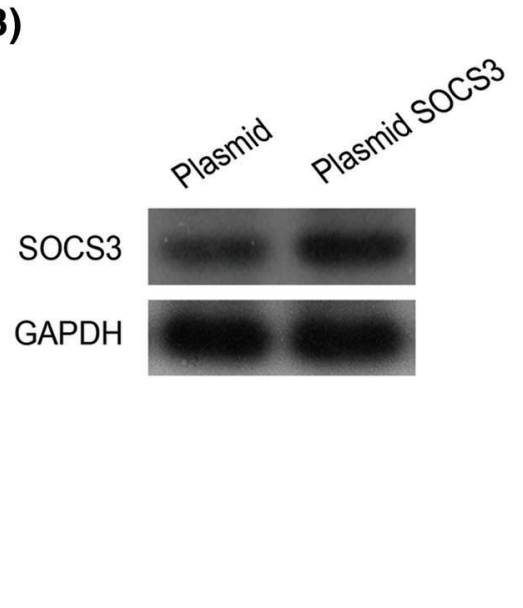

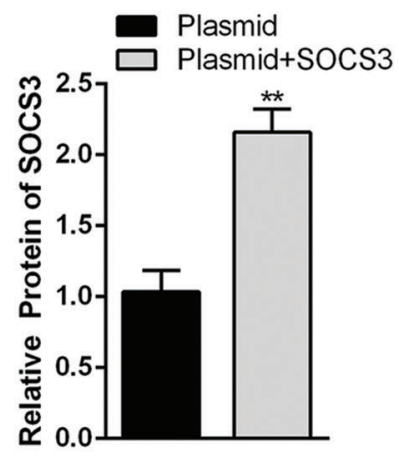

(C)
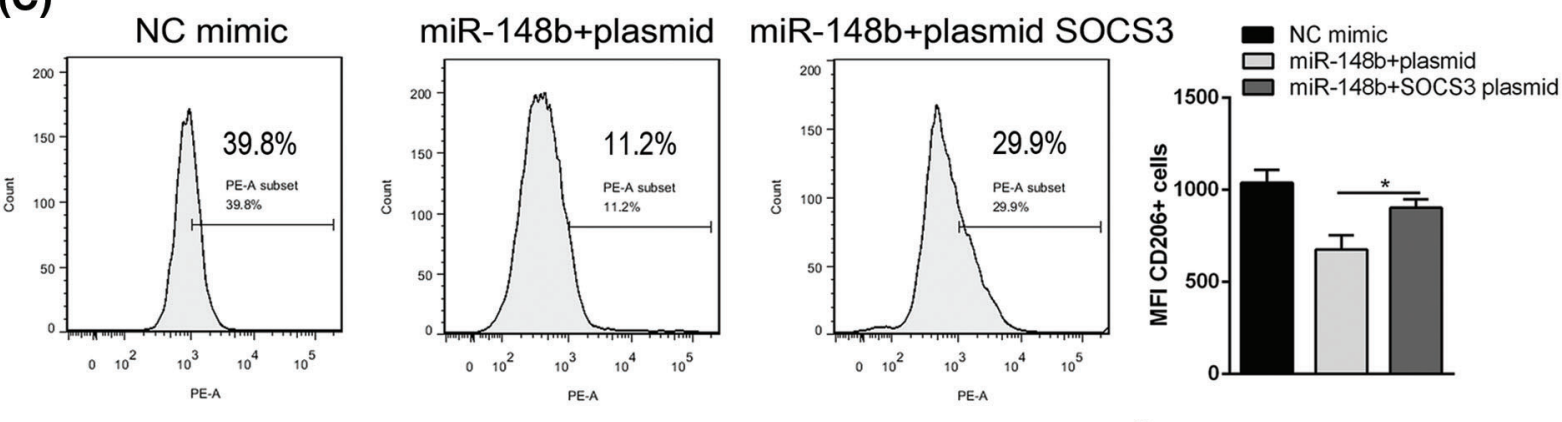

CD206
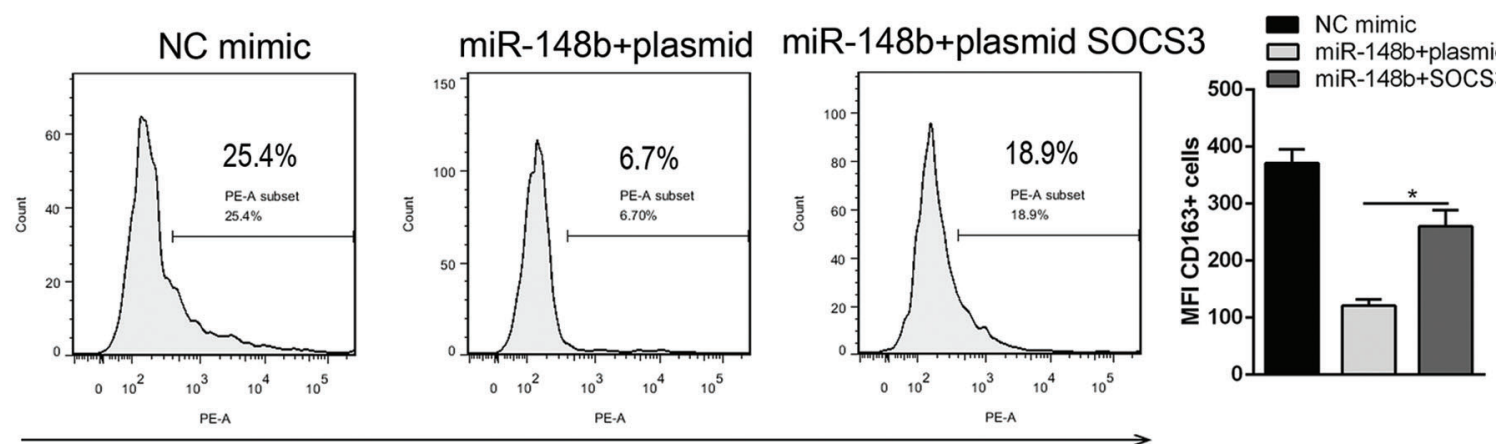

CD163

(D)
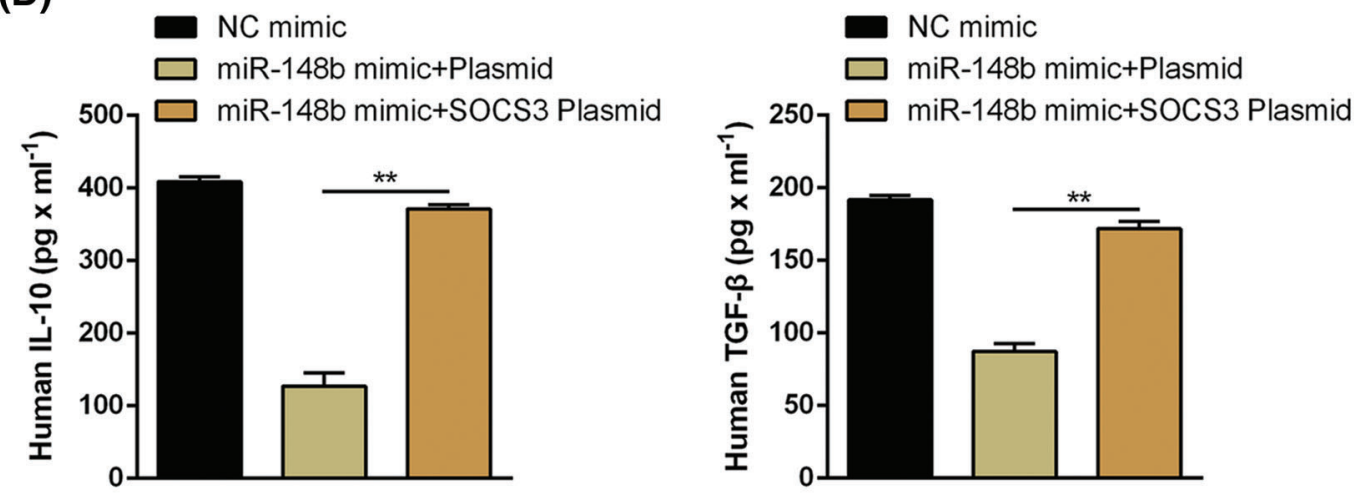

FIGURE 4. (continued) 

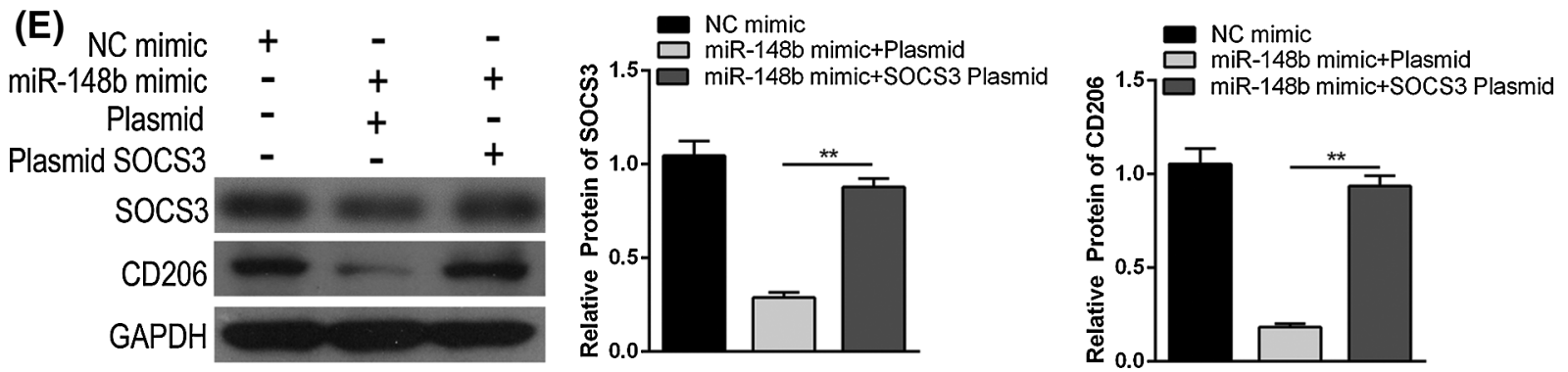

FIGURE 4. miR-148b-3p inhibits macrophage M2 through targeting SOCS3. (A) RT-qPCR was performed to detect the transfection efficiency of miR-148b mimics. (B) Western blot was used to detect the protein levels of SOCS3 in transfected cells. (C-D) Expression of CD206, IL-10 and TGF- $\beta 1$ respectively measured by flow cytometry and ELISA. (E) The protein expression of SOCS3 and CD206 in macrophage detected by Western blot analysis. ${ }^{*} P<0.01,{ }^{*} P<0.05 v$ s. control group.

SOCS3 + miR-148b-3p mimic group compared with control group (Fig. 4D). The above data indicated that upregulation of SOCS3 could reverses the miR-148b-3p mediated inhibition of M2 polarization.

miR-148b-3p inhibited M2 polarization in ITP by facilitating JAK2/STAT3 pathway

To explore the molecular mechanism of miR-148b-3p in macrophage polarization of ITP, we detected phosphorylation of the JAK2/STAT3 signaling pathway by western blot analysis. The results displayed that the expression of SOCS3 and CD206 were noticeably increased, whereas the phosphorylation of JAK2 and STAT3 was accordingly decreased in miR-148b-3p inhibition group. On the contrary, miR-148b-3p mimic promoted the phosphorylation of JAK2/STAT3 and down-regulated macrophages M2 marker (Fig. 5). The above data indicated that the inhibited effect of miR-148b-3p on the M2 polarization depended on its activated function of JAK2/STAT3 in ITP.

\section{Discussion}

Although the current therapeutic regimens have made great progress, a substantial proportion of ITP patients fail to achieve completely clinical remission. Further research of the pathogenesis of ITP is urgently needed to improve patient treatment. ITP is characterized by decreased platelet count and bleeding in the majority of patients. During this process, the imbalance of the immune response and loss of immune tolerance, such as Th1/Th2 imbalance, defective activation of regulatory $\mathrm{T}$ cell (Treg) function, and abnormal M1/M2 macrophages ratio play a crucial role in the pathogenesis of ITP. A previous study has shown that adoptive transfer of M2 macrophages effectively prevented the progression of autoimmune type 1 diabetes in mice, indicating that M2 macrophages play a beneficial role in autoimmune diseases (Parsa et al., 2012). The immunosuppressive action of M2 macrophages is mediated by increased IL-10 and TGF- $\beta$ of production or decreased production of IL-12 and TNF- $\alpha$, further affecting the differentiation and function of $\mathrm{T}$ cells. Furthermore, high-dose dexamethasone or all-transretinoic acid shifted macrophage polarization towards a predominant M2 phenotype, favoring immune tolerance (Feng et al., 2017). These aforementioned studies revealed the prominent role of M2 macrophage polarization in autoimmune inflammatory disease.

Recently, research of miRNAs in ITP is increasing. And dysregulated expression of several miRNAs in ITP has been identified suggesting that miRNAs are regulatory molecules likely involved in the pathogenesis of ITP (Borjigin et al., 2015, Hua et al., 2019). Hence, we explored the potential mechanisms whereby miRNAs influence the progression of ITP, focusing on miR-148b-3p. Preliminary findings of our study revealed that miR-148b-3p was up-regulated and negatively correlated with platelet counts in ITP. Based on the findings of previous studies, the association of miR148a-3p with Class I HLA alleles that play a vital role in platelet clearance, which may be potentially involved in the pathogenesis of ITP and heparin-induced thrombocytopenia (HIT) (Zhou et al., 2015). In addition, the essential role of miR-148b-3p in post-transcriptional gene modulation of the immune system has been explored, which revealed that miR-148b-3p could regulate the expression of PD-L1 on tumor cells and immune suppression via miR-148a-3p downregulation in colorectal cancer (Ashizawa et al., 2019). In the current study, we found that macrophage polarization M2 subtype was promoted while inhibited the progression of ITP through suppression of miR-148b-3p experiments. These aforementioned studies indicated the potential therapeutic target of miR-148b-3p in ITPs.

Additionally, we found that the expression of SOCS3 was decreased in ITPs. SOCS3 is a member of the SOCS family, which plays a prominent role in immune modulation (Kinjyo et al., 2006, Dimitriou et al., 2008). Consistent with our findings, significant down-regulation of SOCS3 have been found in splenic antibody-secreting cells with ITP (Mahévas et al., 2012). Besides, the previous study reported SOCS3 could be downregulated by miR-19b and thereby suppresses the inflammatory response in Crohn's disease (Cheng et al., 2015). It suggests that the likelihood of targeting SOCS3 in therapeutic strategies for the mentioned disorders. To validate this assumption, we overexpressed SOCS3 in macrophages and studied macrophage polarization and function. As expected, the result showed that up-regulation of SOCS3 increased the expression of IL-10, TGF- $\beta$ and CD206, suggesting that SOCS3 transformed macrophage polarization towards a predominant M2 phenotype, favoring immune tolerance. Furthermore, the results of the luciferase activity assay in our study displayed 


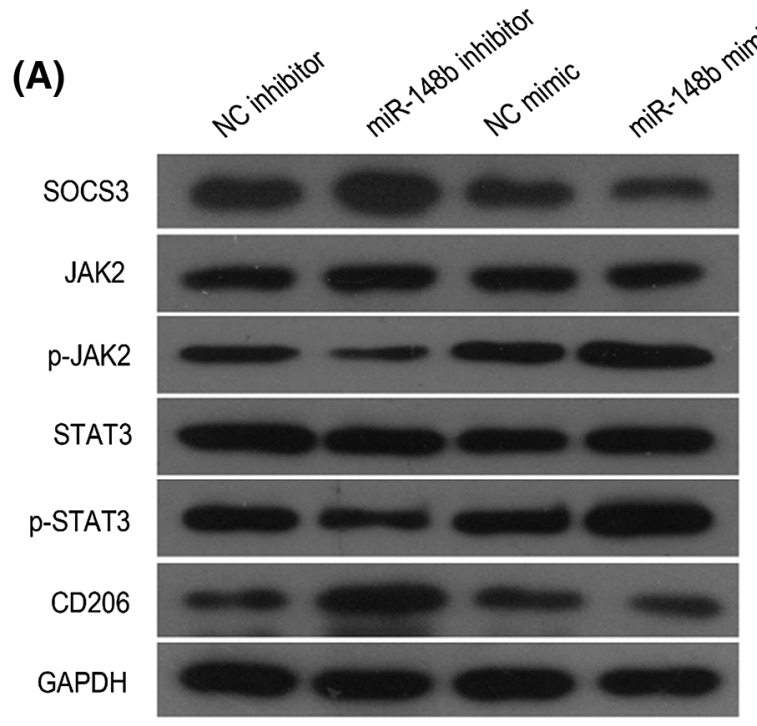

(C)

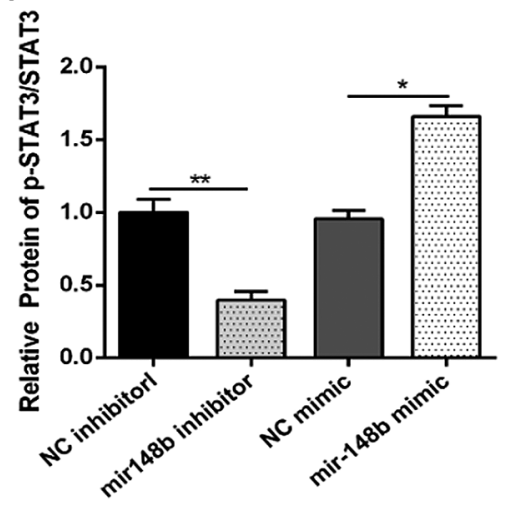

(D)

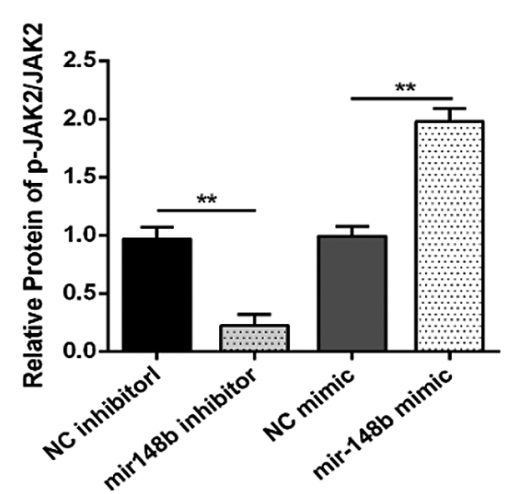

(B)

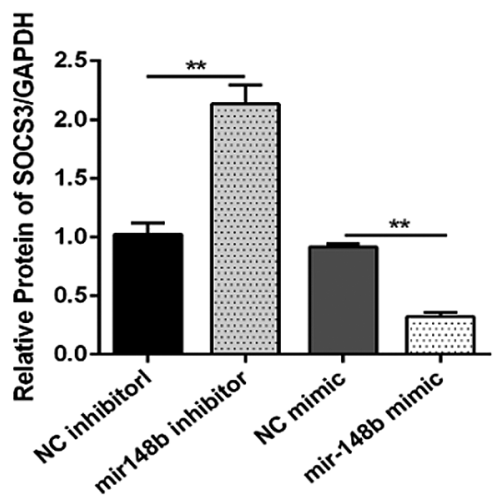

(E)

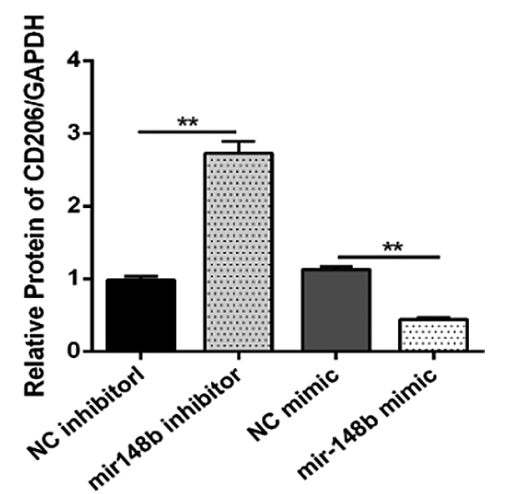

FIGURE 5. miR-148b facilitates JAK2/STAT3 pathway-mediated macrophage M2 polarization by targeting SOCS3. (A) Expression of JAK2/ STAT3 pathway-related proteins, SOCS3 and CD206 were detected by western blot analysis. And the band gray value was analyzed in (B-E). ${ }^{*} P<0.01,{ }^{*} P<0.05$ vs. control group.

that miR-148b-3p could bind to SOCS3. Rescue assay was performed to test the effect of miR-148b mimic reversed by overexpressing SOCS3. This implies that miRNA-148b-3p inhibits macrophage M2 polarization via down-regulating SOCS3 in immune thrombocytopenia.

To explore the mechanism by which miR-148b-3p/ SOCS3 axis mediating the development of ITP, we aimed to find the downstream mechanism. Research shows SOCS3 negatively regulates the JAK2/STAT3 signaling pathway, whereby mediate M2 polarization of macrophages (Tamiya et al., 2011, Shiraishi et al., 2012, Yuan et al., 2014). Therefore, loss-of-function and gain-offunction assays of miR-148b-3p were performed to explore the phosphorylation of the JAK2/STAT3. We found the phosphorylation of the JAK2/STAT3 were inhibited by downregulation of $\mathrm{miR}-148 \mathrm{~b}-3 \mathrm{p}$. Our finding was in accordance with the observations that SOCS3 upregulation, targeted by miRNA-148b-3p, significantly inhibited the JAK2/STAT3 signaling pathway and induced M2 polarization, thus impacting the progression of ITP. All these studies indicated that miR-148b-3p was a potential crucial therapeutic target in ITPs.

In conclusion, our findings provide convincing evidence that suppression of miR-148b-3p promotes macrophage M2 polarization by JAK2/STAT3 pathway and thus inhibit ITP by recovering production of SOCS3, indicating that the regulation of monocytes to M2 polarization may be the potential therapeutic effects for ITP.

Author Contribution: The authors confirm contribution to the paper as follows: study conception and design: Yang Yang, Meiwei $\mathrm{Hu}$; data collection: Lijuan Fu; analysis and interpretation of results: Chunmei Chen; draft manuscript preparation: Yang Yang. All authors reviewed the results and approved the final version of the manuscript.

Availability of Data and Materials: Data supporting this article are details in this manuscript.

Ethics Approval: All protocols for this study was reviewed and approved by The Second Affiliated Hospital of Zhejiang Chinese Medicine University Ethics Committee (2019-KL-049-01).

Funding Statement: This work was supported by grants from the Project of Administration of Traditional Chinese Medicine of Zhejiang Province of China (2022ZB123).

Conflicts of Interest: The authors confirm that there are no conflicts of interest. 


\section{References}

Ashizawa M, Okayama H, Ishigame T, Min AKT, Saito K et al. (2019). miRNA-148a-3p regulates immunosuppression in DNA mismatch repair-deficient colorectal cancer by targeting PD-L1. Molecular Cancer Research 17: 1403-1413.

Batra R, Suh MK, Carson JS, Dale MA, Meisinger TM et al. (2018). IL$1 \beta$ (Interleukin-1 $\beta$ ) and TNF- $\alpha$ (Tumor Necrosis Factor- $\alpha$ ) impact abdominal aortic aneurysm formation by differential effects on macrophage polarization. Arteriosclerosis, Thrombosis, and Vascular Biology 38: 457-463.

Borjigin M, Huo W, Gong C, Zhang G, Li M et al. (2015). Profiling of miRNA expression in immune thrombocytopenia patients before and after Qishunbaolier (QSBLE) treatment. Biomedicine \& Pharmacotherapy 75: 196-204.

Chang Y, Chen X, Tian Y, Gao X, Liu Z et al. (2020). Downregulation of microRNA-155-5p prevents immune thrombocytopenia by promoting macrophage M2 polarization via the SOCS1dependent PD1/PDL1 pathway. Life Sciences 257: 118057.

Cheng X, Zhang X, Su J, Zhang Y, Zhou W et al. (2015). miR-19b downregulates intestinal SOCS3 to reduce intestinal inflammation in Crohn's disease. Scientific Reports 5: 10397.

Chi X, Ding B, Zhang L, Zhang J, Wang J et al. (2019). lncRNA GAS5 promotes M1 macrophage polarization via miR-455-5p/ SOCS3 pathway in childhood pneumonia. Journal of Cellular Physiology 234: 13242-13251.

Cines DB, Bussel JB, Liebman HA, Luning Prak ET (2009). The ITP syndrome: Pathogenic and clinical diversity. Blood 113: 6511-6521.

Cui J, Zhang F, Cao W, Wang Y, Liu J et al. (2018). Erythropoietin alleviates hyperglycaemia-associated inflammation by regulating macrophage polarization via the JAK2/STAT3 signalling pathway. Molecular Immunology 101: 221-228.

Dimitriou ID, Clemenza L, Scotter AJ, Chen G, Guerra FM et al. (2008). Putting out the fire: Coordinated suppression of the innate and adaptive immune systems by SOCS1 and SOCS3 proteins. Immunological Reviews 224: 265-283.

Feng Q, Xu M, Yu Y, Hou Y, Mi X et al. (2017). High-dose dexamethasone or all-trans-retinoic acid restores the balance of macrophages towards M2 in immune thrombocytopenia. Journal of Thrombosis and Haemostasis 15: 1845-1858.

Hua M, Li J, Wang C, Shao L, Hou M et al. (2019). Aberrant expression of microRNA in CD4+ cells contributes to Th17/Treg imbalance in primary immune thrombocytopenia. Thrombosis Research 177: 70-78.

Joshi AD, Oak SR, Hartigan AJ, Finn WG, Kunkel SL et al. (2010). Interleukin-33 contributes to both M1 and M2 chemokine marker expression in human macrophages. BMC Immunology 11: 52.

Kinjyo I, Inoue H, Hamano S, Fukuyama S, Yoshimura T et al. (2006). Loss of SOCS3 in T helper cells resulted in reduced immune responses and hyperproduction of interleukin 10 and transforming growth factor- $\beta 1$. Journal of Experimental Medicine 203: 1021-1031.

Li JQ, Hu SY, Wang ZY, Lin J, Jian S et al. (2016). Long non-coding RNA MEG3 inhibits microRNA-125a-5p expression and induces immune imbalance of Treg/Th17 in immune thrombocytopenic purpura. Biomedicine \& Pharmacotherapy 83: 905-911.

Liu J, Qiu P, Qin J, Wu X, Wang X et al. (2020). Allogeneic adiposederived stem cells promote ischemic muscle repair by inducing M2 macrophage polarization via the HIF-1 $\alpha /$ IL-10 pathway. Stem Cells 38: 1307-1320.

Mahévas M, Patin P, Huetz F, Descatoire M, Cagnard N et al. (2012). B cell depletion in immune thrombocytopenia reveals splenic longlived plasma cells. Journal of Clinical Investigation 123: 432-442.

Park E, Jung H, Yang H, Yoo M, Kim C, Kim K (2007). Optimized THP-1 differentiation is required for the detection of responses to weak stimuli. Inflammation Research 56: 45-50.

Parsa R, Andresen P, Gillett A, Mia S, Zhang XM et al. (2012). Adoptive transfer of immunomodulatory M2 macrophages prevents type 1 diabetes in NOD mice. Diabetes 61: 2881-2892.

Pauley KM, Cha S, Chan EK (2009). MicroRNA in autoimmunity and autoimmune diseases. Journal of Autoimmunity 32: 189-194.

Qian BH, Ye X, Zhang L, Sun Y, Zhang JR et al. (2015). Increased miR155 expression in peripheral blood mononuclear cells of primary immune thrombocytopenia patients was correlated with serum cytokine profiles. Acta Haematologica 133: 257-263.

Shiraishi D, Fujiwara Y, Komohara Y, Mizuta H, Takeya M (2012). Glucagon-like peptide-1 (GLP-1) induces M2 polarization of human macrophages via STAT3 activation. Biochemical and Biophysical Research Communications 425: 304-308.

Singh RP, Massachi I, Manickavel S, Singh S, Rao NP et al. (2013). The role of miRNA in inflammation and autoimmunity. Autoimmunity Reviews 12: 1160-1165.

Tamiya T, Kashiwagi I, Takahashi R, Yasukawa H, Yoshimura A (2011). Suppressors of cytokine signaling (SOCS) proteins and JAK/STAT pathways: Regulation of T-cell inflammation by SOCS1 and SOCS3. Arteriosclerosis, Thrombosis, and Vascular Biology 31: 980-985.

Wang N, Liang H, Zen K (2014). Molecular mechanisms that influence the macrophage M1-M2 polarization balance. Frontiers in Immunology 5: 614.

Yu S, Liu C, Li L, Tian T, Wang M et al. (2015). Inactivation of Notch signaling reverses the Th17/Treg imbalance in cells from patients with immune thrombocytopenia. Laboratory Investigation 95: 157-167.

Yuan F, Fu X, Shi H, Chen G, Dong P et al. (2014). Induction of murine macrophage M2 polarization by cigarette smoke extract via the JAK2/STAT3 pathway. PLoS One 9: e107063.

Zhou Y, Abraham S, Andre P, Edelstein LC, Shaw CA et al. (2015). Anti-miR-148a regulates platelet Fc $\gamma$ RIIA signaling and decreases thrombosis in vivo in mice. Blood, The Journal of the American Society of Hematology 126: 2871-2881. 


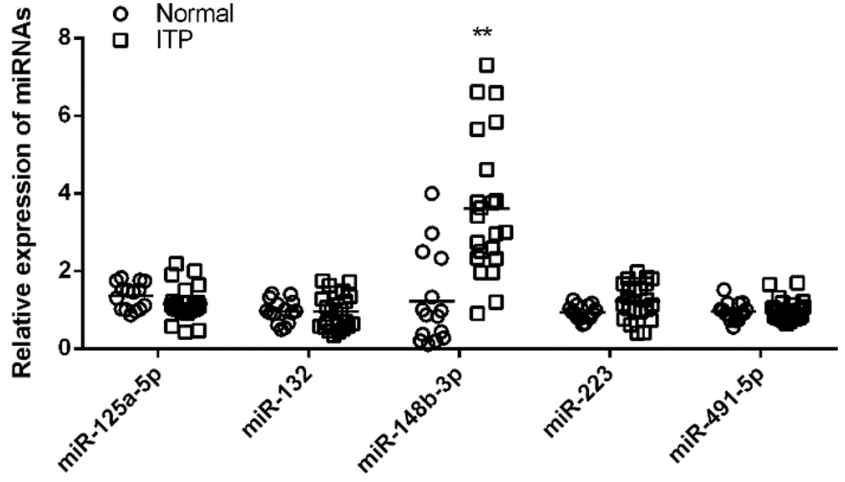

FIGURE S1. Relative expression of miRNAs expression in ITP patients $(\mathrm{n}=22)$ and controls $(\mathrm{n}=15)$. miR-148b-3p was significantly upregulated in ITPs detected by RT-qPCR.

\section{SUPPLEMENTAL TABLE 1}

ITP patients information

\begin{tabular}{|c|c|c|c|}
\hline Patient No. & Sex/Age (year) & Bleeding symptoms & Platelet count $\left(\times 10^{9} / \mathrm{L}\right)$ \\
\hline 1 & $\mathrm{~F} / 54$ & $\mathrm{EC}$ & 18.3 \\
\hline 2 & $\mathrm{M} / 22$ & PT & 22.3 \\
\hline 3 & $\mathrm{M} / 32$ & PT, EC & 18.5 \\
\hline 4 & $\mathrm{~F} / 51$ & PT, EC & 12.2 \\
\hline 5 & $\mathrm{~F} / 38$ & None, GUH & 17.3 \\
\hline 6 & $\mathrm{~F} / 48$ & $\mathrm{GH}, \mathrm{EC}$ & 13.4 \\
\hline 7 & $\mathrm{~F} / 33$ & None, GUH & 24.3 \\
\hline 8 & $\mathrm{M} / 62$ & None & 17.1 \\
\hline 9 & $\mathrm{M} / 31$ & PT & 12.2 \\
\hline 10 & $\mathrm{M} / 51$ & None & 11.5 \\
\hline 11 & $\mathrm{M} / 39$ & EC, EP & 17.6 \\
\hline 12 & $\mathrm{~F} / 73$ & None & 9.1 \\
\hline 13 & $\mathrm{M} / 23$ & $\mathrm{EC}$ & 24.4 \\
\hline 14 & $\mathrm{~F} / 49$ & PT, EP & 21.8 \\
\hline 15 & $\mathrm{M} / 57$ & $\mathrm{PT}$ & 17.6 \\
\hline 16 & $\mathrm{~F} / 67$ & None & 15.7 \\
\hline 17 & $\mathrm{M} / 42$ & PT, EP & 14.3 \\
\hline 18 & $\mathrm{M} / 54$ & None & 15.6 \\
\hline 19 & $\mathrm{M} / 32$ & $\mathrm{EC}$ & 18.5 \\
\hline 20 & $\mathrm{~F} / 28$ & EC, GUH & 12.4 \\
\hline 21 & $\mathrm{M} / 48$ & None & 22.3 \\
\hline 22 & $\mathrm{M} / 68$ & None & 28.6 \\
\hline
\end{tabular}

